\title{
Broadband output windows for THz gyro-TWAs
}

\author{
Craig R. Donaldson, Liang Zhang and Wenlong He \\ Department of Physics, SUPA, University of Strathclyde, Glasgow, Scotland, UK, G4 0NG
}

\begin{abstract}
This paper presents the design, simulation and technical considerations of output windows, based on the multilayer type, for THz gyro-TWAs. Such windows can achieve very low reflection levels over a broadband frequency range when the thickness of the dielectric layers are properly matched. The optimized window, in this design, was numerically calculated to achieve a passband of over $7 \%$ with a reflection of less than $30 \mathrm{~dB}$ and transmission loss of better than $-0.3 \mathrm{~dB}$.
\end{abstract}

\section{INTRODUCTION}

$\mathrm{T}$ HE gyro-TWA has potential to generate high power broadband radiation up to the $\mathrm{THz}$ frequency range. Such amplifiers benefit from having an enlarged interaction region in comparison with conventional TWT-like amplifiers. Thus, they do not suffer the power handling problems of their counterparts. The already wide bandwidth of the gyro-TWAs can be further enhanced if using a helically corrugated interaction region (HCIR) [1,2]. In the W-band frequency range two gyro-devices based on the HCIR in conjunction with a cusp electron gun $[3,4]$ have been realized. Initially a gyrotron backward wave oscillator was demonstrated to have $13 \mathrm{~kW}$ output power over a frequency range of 88 to 102.5 $\mathrm{GHz}$ [5]. More recently, a gyro-TWA showed a $3.4 \mathrm{~kW}$ output over 91 to $96.5 \mathrm{GHz}$, this range is stunted by the limitation of the driving source [6,7]. Currently, a gyro-TWA operating centrally at $372 \mathrm{GHz}$, with $5 \%$ bandwidth, is being developed. Similar to the aforementioned devices a HCIR will be used and the beam-wave interaction will be driven by an axisencircling electron beam generated by a cusp electron gun $[8,9]$ with thermionic cathode. Many components are required in the system including an input coupler [10], interaction region [1] and both input [11] and output windows [12]. This paper considers the design of the output window which will act to separate the UHV inside the experiment from the atmosphere surrounding it. The window will also be required to be transparent to the passing $\mathrm{THz}$ radiation, with a reflection of less than $-30 \mathrm{~dB}$.

While there are a few window designs that can meet the requirements for this amplifier, such as the multilayer [12,13], pillbox [11] and Brewster [14], the multilayer window was chose due, in part, to the authors experience in realizing such windows. In this configuration the widest passband is achieved when a sandwich of multiple (3, 5, 7, and so on) dielectric layers with both the thickness and permittivity of the layers optimized and matched. In this design five layers give sufficient bandwidth and so this can be achieved by a singular dielectric disc which is flanked by two matching dielectric discs, spaced by a small vacuum/air gap, as shown in Fig. 1.

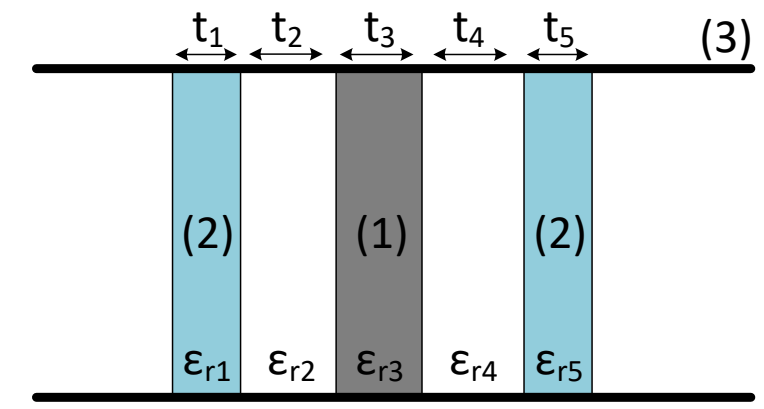

Fig. 1 The multilayer window schematic of its configuration.

The central disc (1) was chose to be Alumina, $99.4 \%$ pure, as this can be readily vacuum brazed, is mechanically strong and compatible with the operation of a thermionic cathode. The relative dielectric constant of the Alumina is $\varepsilon_{r 3}=9.7$ The matching dielectric discs (2) are chose to be Quartz which has a relative dielectric constant of $\varepsilon_{r 1}=\varepsilon_{r 5}=3.75$. These are housed in an OFHC Cu waveguide (3).

\section{NUMERICAL OPTIMIZATION}

Optimization of the window structure can be conveniently made through numerical calculation using the mode matching method. A single solution can be obtained in seconds while a full 3D solver will take many minutes. Initially the window structure was calculated while the diameters of the dielectric layers were all equal. The result from this shows only the reflection from the dielectric layers rather than those caused by discontinuities in the waveguide structure. The number of free parameters in the optimization was also reduced leading to a faster optimization process. In realization, however, the Quartz discs should be precisely located and secured which would inevitably involve the modifications to the window structure which would undoubtedly lead to unwanted reflections. However, the effect of the waveguide discontinuities on the reflection can be reduced through the use of the Gaussian-like $\mathrm{HE}_{11}$ mode, which concentrates the electromagnetic energy in the center of the waveguide. To achieve this mode converters in the form of corrugated [15] or smoothly profiled horn [16] can be used to converter normal $\mathrm{TE}_{11}$ mode before the window. 


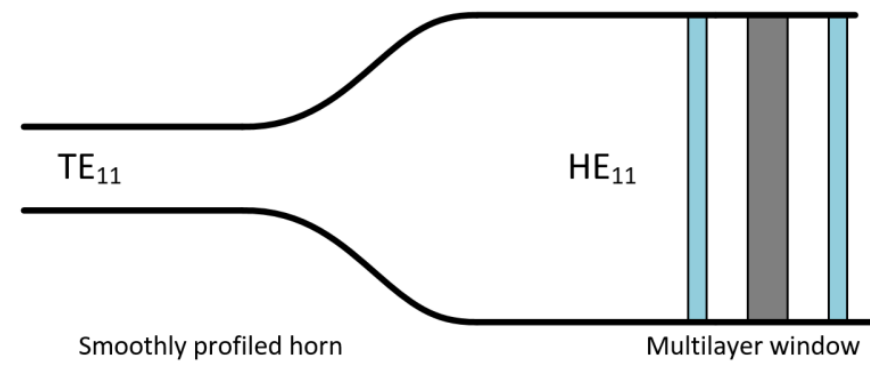

Fig. 2 Schematic of the multilayer window and mode converting smoothly profiled horn.

The multilayer window was realized at W-band [12]. The measured reflection is shown in Fig. 3 and a better than $-30 \mathrm{~dB}$ reflection was achieved for a passband of 90-100 $\mathrm{GHz}$. Furthermore, the measurement was in good agreement with the numerical calculation which gives confidence to the design methodology.

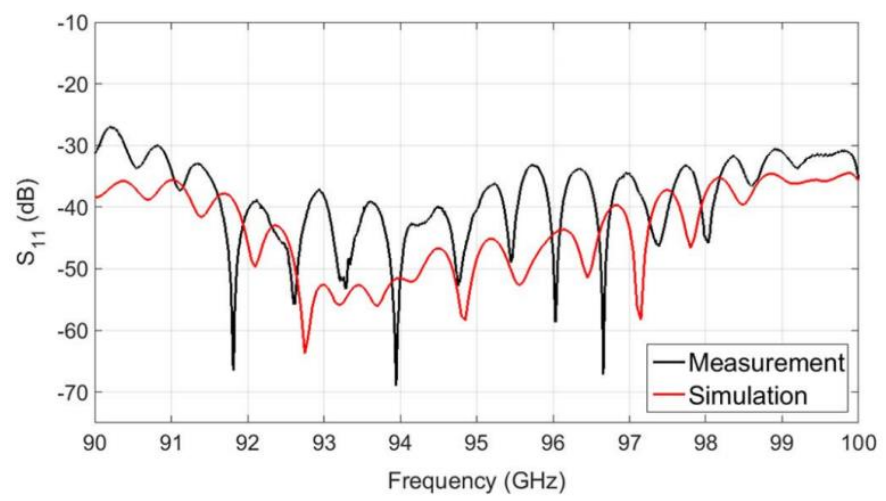

Fig. 3 The measured and simulated multilayer window at W-band frequencies.

The optimized window shows that a reflection of less than -30 $\mathrm{dB}$ can be achieved over a frequency range of 360 to 384 $\mathrm{GHz}$, as shown in Fig. 4. In this case the thickness of the central dielectric disc (T3) was $0.809 \mathrm{~mm}$, for the Quartz discs $\mathrm{T} 1=\mathrm{T} 5=0.255 \mathrm{~mm}$ and the vacuum gaps $\mathrm{T} 2=\mathrm{T} 4=0.056$ $\mathrm{mm}$. Both this multilayer window and the smoothly profiled horn will be constructed and microwave properties measured on a vector network analyzer.

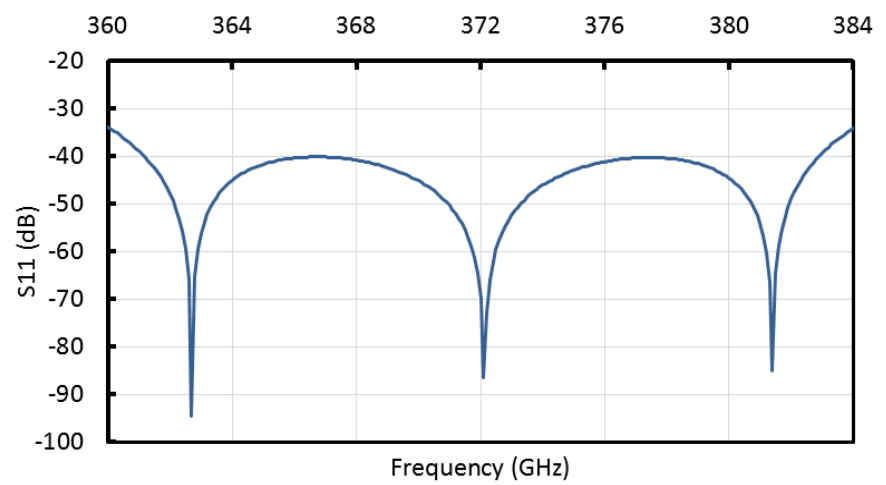

Fig. 4 The optimized multilayer window reflection calculation.

\section{REFERENCES}

[1] C.R. Donaldson, L. Zhang, M. Beardsley, et al., "CNC machined helically corrugated interaction region for a $\mathrm{THz}$ gyrotron traveling wave amplifier," IEEE Trans. THz Sci. Techn., 8, pp.85-89, 2017.

[2] L. Zhang, W. He, K. Ronald, et al., "Multi-mode coupling wave theory for helically corrugated waveguide," IEEE Trans. Microw. Theory Techn., 60, pp. $1-7,2012$.

[3] L. Zhang, W. He, C. R. Donaldson, and A. W, Cross, "Investigation on the optimal magnetic field of a cusp electron gun for a W-band gyro-TWA," Phys. Plasmas, 25, 053104, 2018.

[4] L. Zhang, C. R. Donaldson, and W. He, "Optimization of a triode-type cusp electron gun for a W-band gyro-TWA," Phys. Plasmas, 25, 043120, 2018.

[5] W. He, C. R. Donaldson, L. Zhang, et al., "High power wideband gyrotron backward wave oscillator operating towards the terahertz region," Phys. Rev. Lett., 110, 165101, 2013.

[6] W. He, C.R. Donaldson, L. Zhang, et al., "Broadband amplification of low-terahertz signals using axis-encircling electrons in a helically corrugated interaction region," Phys. Rev. Lett., 119, 196801, 2017.

[7] L. Zhang, C. R. Donaldson, P. Cain, et al., "Amplification of frequencyswept signals in a W-band gyrotron travelling wave amplifier," IEEE Trans. Electron Dev. Lett., DOI: 10.1109/LED.2018.2836868.

[8] W. He, C. G. Whyte, E. G. Rafferty, et al., "Axis-encircling electron beam generation using a smooth magnetic cusp for gyrodevices," Appl. Phys. Lett., 93, 121501, 2008.

[9] C.R. Donaldson, W. He, A.W. Cross, et al., "A cusp electron gun for millimeter wave gyrodevices," Appl. Phys. Lett., 96, 141501, 2010.

[10] L. Zhang, W. He, C.R. Donaldson, et al., "Design and measurement of a broadbad sidewall coupler for a W-band gyro-TWA," IEEE Trans. Microw. Theory Techn., 63, pp. 3183-3190, 2015.

[11] L. Zhang, C. R. Donaldson, A. W. Cross, and W. He, et al., "A pillbox window with impedance matching sections for a W-band gyro-TWA," IEEE Trans, Electron Dev. Lett., DOI: 10.1109/LED.2018.2834859.

[12] C.R. Donaldson, P. McElhinney, L. Zhang, and W. He, "Wide-band $\mathrm{HE}_{11}$ mode terahertz wave windows for gyro-amplifiers," IEEE THz Sci. Techn., 6 , pp. 108-112, 2016

[13] C.R. Donaldson, W. He, L. Zhang, and A.W. Cross, "A W-band mutlilayer microwave window for pulsed operation of gyro-devices," IEEE Micro. Wireless Compon. Lett., 23, pp. 237-239, 2013.

[14] Y. Zhang, L. Zhang, W. He, et al., "Design and measurement of a Wband Brewster window," IEEE Mirow. Wireless Comp. Lett., 25, pp. 826-828, 2015.

[15] P. McElhinney, C. R. Donaldson, L. Zhang and W. He., "A high directivity broadband corrugated horn for W-band gyro-devices," IEEE Trans. Antennas Propg., 61, pp. 1453-1456, 2013.

[16] L. Zhang, W. He, C. R. Donaldson, et al., "Optimization and measurement of a smoothly profiled horn for a W-band gyro-TWA," IEEE Trans. Electron Dev., 64, pp. 2665-2669, 2017. 